\title{
When Do Ring-Enhancing Brain Lesions Need to Be Biopsied, and Should They Be Treated Empirically First?
}

\author{
Nadia Khosrodad ${ }^{1}$, Justin Khine ${ }^{2}$, Jeffrey Maclean ${ }^{3}$, Fnu Abhishek ${ }^{4}$ \\ ${ }^{1}$ Department of Internal Medicine, St. Joseph Mercy Oakland Hospital, Pontiac, MI, USA \\ ${ }^{2}$ Clinical Teaching Fellow, Ross University, Miramar, FL, USA \\ ${ }^{3}$ Department of Radiology, St. Joseph Mercy Oakland Hospital, Pontiac, MI, USA \\ ${ }^{4}$ Department of Neurology, St. Joseph Mercy Oakland Hospital, Pontiac, MI, USA
}

Received: 05/02/2019

Accepted: $18 / 03 / 2019$

Published: $10 / 04 / 2019$

How to cite this article: Khosrodad N, Khine J, Maclean J, Abhishek F. When do ring-enhacing brain lesions need to be biopsed, and shoud they be treated empirically first? EJCRIM 2019;6: doi:10.12890/2019_001068.

Conflicts of Interests: The Authors declare that there are no competing interests.

This article is licensed under a Commons Attribution Non-Commercial 4.0 License

\section{ABSTRACT}

Other than acute cerebrovascular accidents, multiple ring-enhancing lesions are among the most common lesions encountered in neuroimaging. We herein describe the case of a 63-year-old diabetic man presenting with altered mental status, hyperglycaemia and community-acquired pneumonia who was found to have two ring-enhancing lesions involving the left frontal lobe and left basal ganglia. The lesions were biopsied to reveal positive fungal cultures and toxoplasma cysts. RPR titres returned reactive for non-treponemal antibodies and a suppressed CD4 count was found without evidence of HIV infection.

\section{LEARNING POINTS}

- An approach is discussed that will direct clinicians to decide whether to treat ring-enhancing brain lesions empirically or biopsy them first.

\section{KEYWORDS}

Neuroimaging, abscess, immunocompromised, toxoplasmosis, PCNSL, idiopathic CD4 leukocytopenia

\section{INTRODUCTION}

The aetiologies of ring-enhancing lesions range from neoplastic, inflammatory, infectious and autoimmune diseases to vascular causes. Radiographic imaging, consideration of aetiologies, and a comprehensive clinical picture of the patient with attention to immunocompetency are important considerations. These will guide the clinicians' decision to perform a biopsy and culture of the lesion, and to initiate appropriate antimicrobial therapy in a timely manner ${ }^{[1,2]}$. A reasonable clinical suspicion of an infectious aetiology guides empirical treatment, but when should a biopsy be performed? Should it be before treatment or after no improvement is seen following initiation of empirical antimicrobial therapy?

\section{CASE REPORT}

A 63-year-old African American man with a medical history of diabetes presented to the emergency department with altered mental status. He was found confused in his car and emergency medical services were called for confusion as well as unequal pupil sizes. The patient was able to report that he had experienced subjective fevers accompanied by cough and chills at home for several days. Vital signs were stable on presentation. Physical examination was significant for anisocoria, dysarthria, right upper and lower extremity weakness, and right facial 


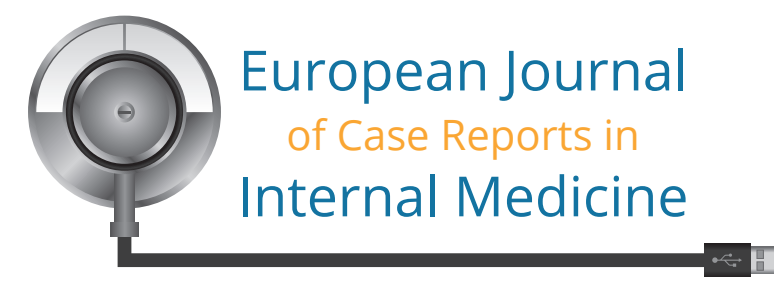

droop. Initial laboratory findings were remarkable for a blood glucose level of $376 \mathrm{mg} / \mathrm{dl}$ and an absence of leucocytosis. A chest x-ray revealed left lower lobe infiltrates suspicious for pneumonia (Fig. 1).

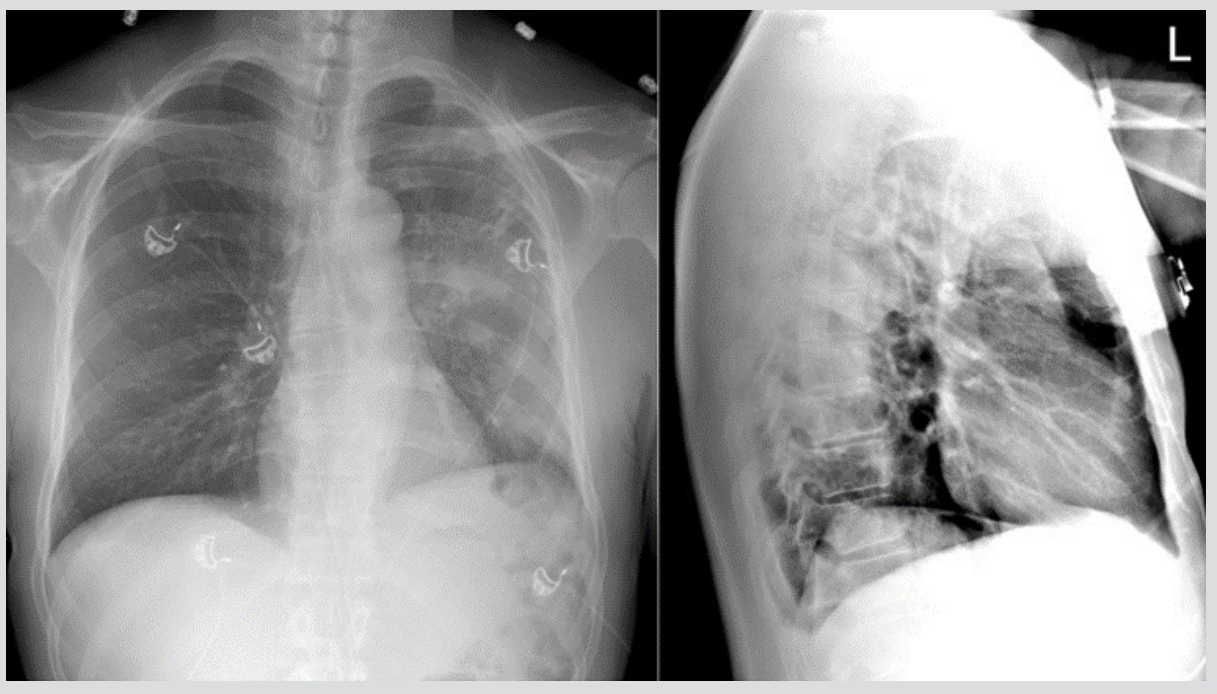

Figure 1. Postero-anterior (left) and lateral (right) chest $x$-ray showing left lower lobe infiltrate

CT's of the abdomen, pelvis, and chest revealed consolidative changes in left lower lung fields, a benign hepatic hemangioma, and an adrenal adenoma. CT-guided biopsy of the lung mass revealed non-malignant findings consistent with inflammatory changes that support the community-acquired pneumonia. Empirical treatment was started with ceftriaxone and azithromycin for community-acquired pneumonia. Computed tomography (CT) of the head without contrast showed asymmetric hypodensities in the left thalamus. MRI of the brain revealed a ring-enhancing lesion in the left frontal lobe, and a ring-enhancing lesion in the left basal ganglia with extension and abutment of the left pons with diffuse and extensive vasogenic oedema causing a midline shift and $3 \mathrm{~mm}$ mass effect into the left ventricle (Figs. 2-4).

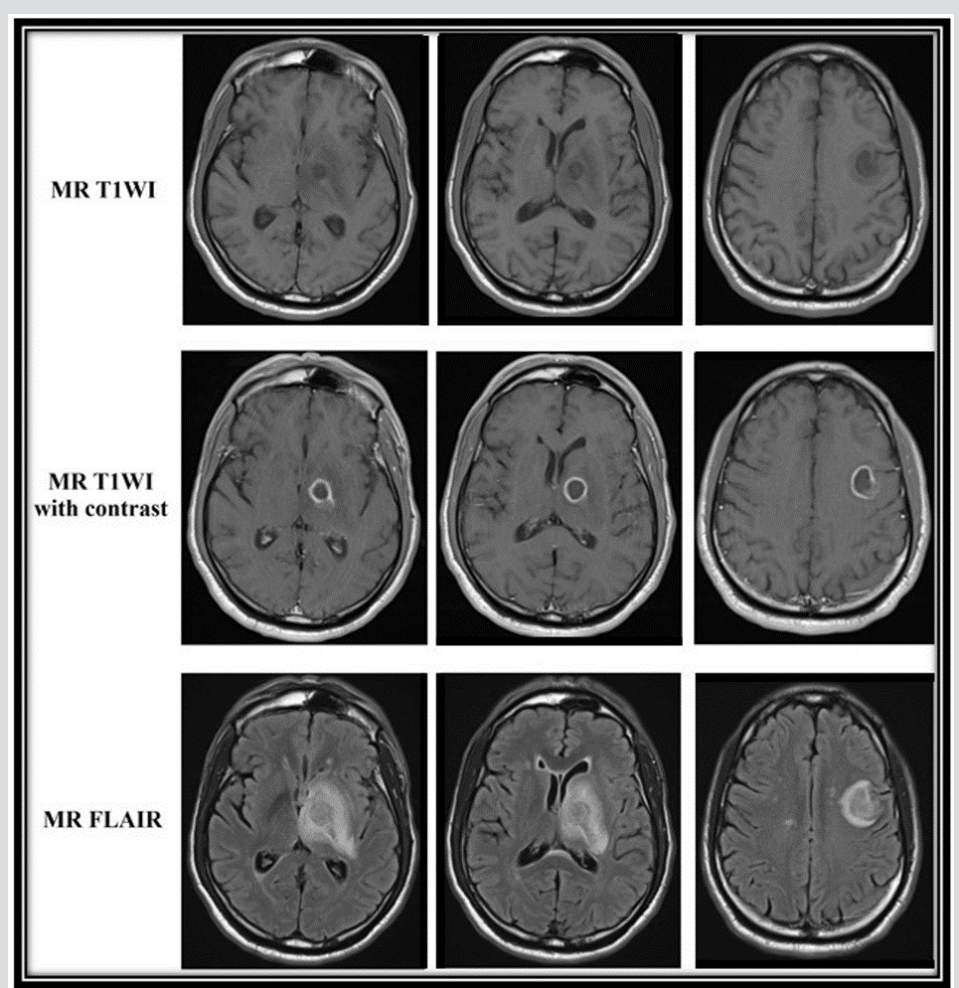

Figure 2.Axial MR images arranged by sequence and synchronized by slice through the ring-enhancing lesions in the left basal ganglia (two left columns) and the left frontal lobe (right column) 


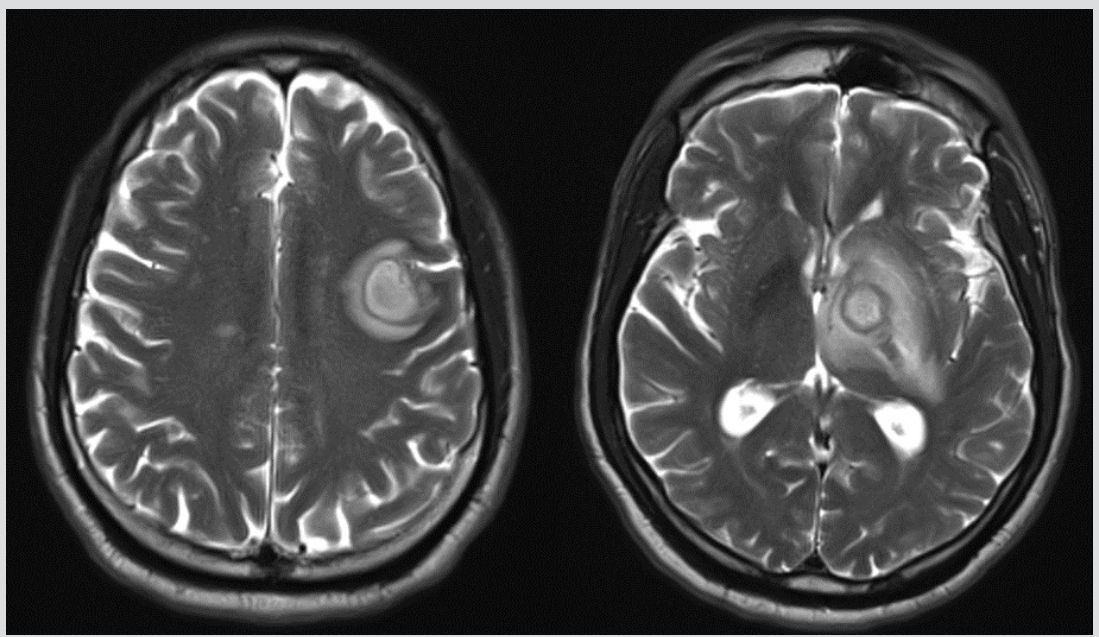

Figure 3. Axial MR T2WI of a $1.6 \times 2.1 \times 1.1 \mathrm{~cm}$ ring-enhancing lesion in the left frontal lobe (left) and a

$1.7 \times 1.6 \times 3.4 \mathrm{~cm}$ ring-enhancing lesion in the left basal ganglia with extension and abutment of the left pons (right)

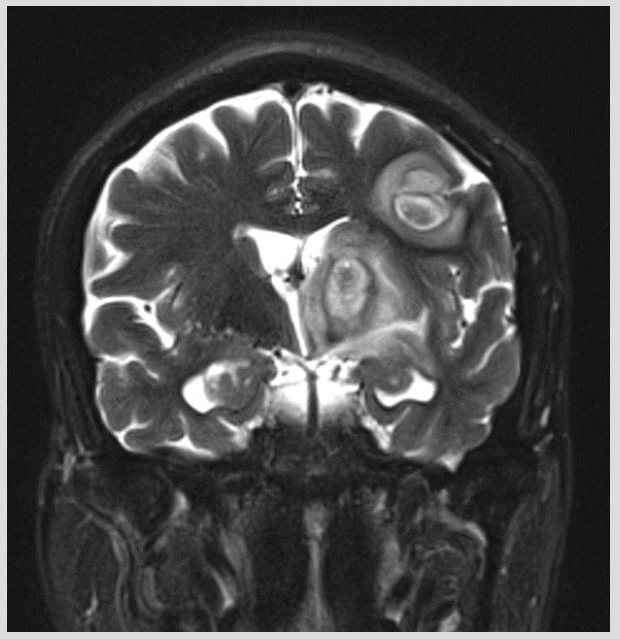

Figure 4. Coronal MR T2WI of both lesions showing mass effect and midline shift

The patient was started on vancomycin, ceftriaxone, ampicillin, acyclovir, sulfamethoxazole/trimethoprim, metronidazole, Keppra for seizure prophylaxis, and dexamethasone to decrease cerebral oedema.

The patient underwent extensive investigation for infectious causes, which only revealed positive results for syphilis and toxoplasma IgGs. Of note, flow cytometry showed a CD4 count of 204 cells $/ \mathrm{mm}^{3}$ and tests were negative for HIV, TB, cryptococcus, hepatitis B, hepatitis $\mathrm{C}$, histoplasmosis and legionella. Primary cancers and brain metastases were considered, but CT imaging did not reveal any evidence of malignancies. In light of the above results, the patient was started on penicillin for syphilis and remained on Keppra given the extensive nature of the ring-enhancing lesions.

A brain biopsy was performed as the presence of space-occupying lesions made a lumbar puncture inadvisable. Results were compatible with an inflammatory/infectious process (Fig. 5a), having one focus resembling a toxoplasma cyst (Fig. 5b,c), and cultures were notable for one colony of Aspergillus fumigatus isolated from one of three plates. Additional stains for GMS, SV40, Twort, Ziehl-Neelsen, FITE, CMV, HSV $1 / 2$ and $V Z V$ were negative. At this point, due to the presumed diagnosis of cerebral aspergillosis and toxoplasmosis, as well as the confirmed diagnosis of syphilis, the patient's antimicrobial treatments were adjusted to only include voriconazole, sulfamethoxazole/trimethoprim and penicillin G.

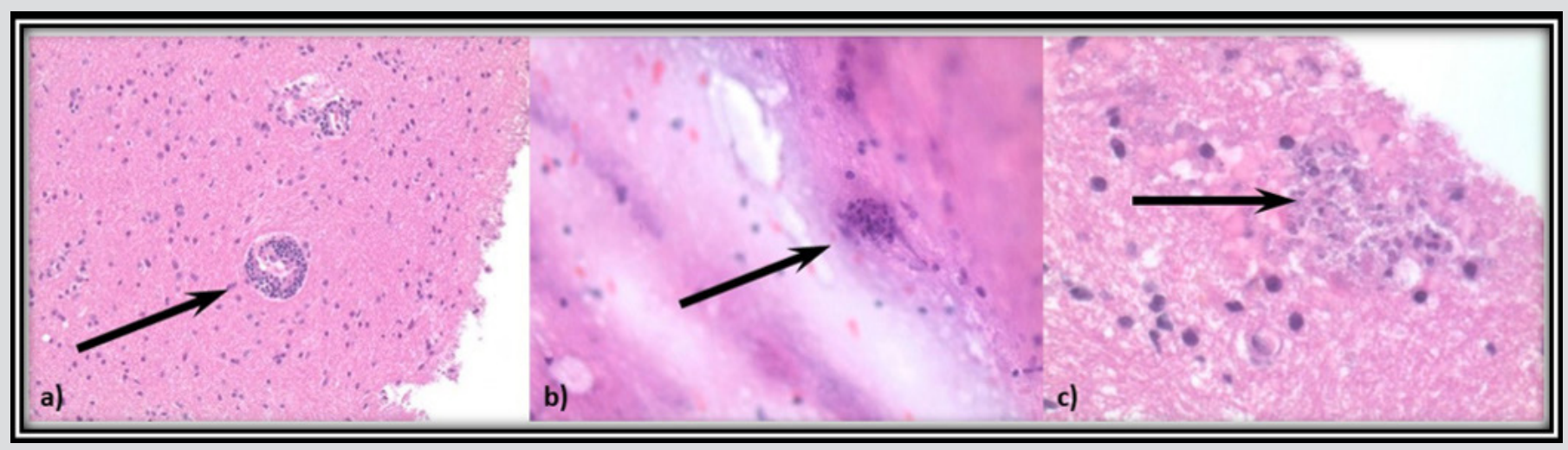

Figure 5. a) Lymphocytic cuffing around vessels from biopsied brain tissue. b) Toxoplasma cyst found in a sample of brain biopsy tissue. c) Areas suspicious for anothertoxoplasma cyst

Haematological malignancies were considered, but ANA, c-ANCA, p-ANCA, Smith antibodies and other extractable nuclear antigens were negative. Repeat MRI of the brain 14 days later redemonstrated the ring-enhancing lesions, which were now smaller in size with decreases 
in the degree of enhancement and the surrounding oedema. Courses of sulfamethoxazole/trimethoprim, penicillin and steroids were completed and the patient was discharged on 3 months of voriconazole.

\section{DISCUSSION}

In this case report, a CD4 lymphocytopenic patient without HIV infection or a history of organ transplantation was found to have brain abscesses that were biopsied to yield toxoplasmosis and Aspergillus species. Toxoplasmosis is an opportunist and ubiquitous infection and one of the most common infectious aetiologies of brain abscesses in immunocompromised hosts. A significantly more uncommon cause of brain abscess is Aspergillus, a ubiquitous fungus inhaled as airborne spores and producing septated hyphae ${ }^{[3]}$. Aspergillus infections are extremely rare in the CNS of immunocompetent hosts and carry a high mortality rate of $88-90 \%[3,4]$.

The differential diagnoses that should be considered in an immunocompromised host include primary central nervous system lymphoma (PCNSL) and toxoplasmosis when there is evidence of ring-enhancing lesions; their radiological differentiation is subtle but can direct early treatment. Typical imaging of toxoplasmosis, and other bacterial or viral abscesses, displays signs of central homogenous hypodensity indicating central necrosis ${ }^{[1]}$. PCNSL presents on MR imaging as a uniformly homogeneous moderate-to-intense signal enhancement.

This is sometimes also seen in rapidly growing brain tumours such as glioblastomas. There are other definitive tests, such as biopsy of the lesion or DNA PCR from cerebrospinal fluid for causative infections, that offer a more precise means of identification. Often the decision to perform a biopsy is only considered after initial empirical treatment has failed. Early biopsy, however, has the potential to reveal causative agents, leading to faster initiation of definitive treatment. This has the potential to reduce the duration of hospitalization and costs associated with pursuing other differential diagnoses. However, clinicians must consider the availability of resources in their facility for timely brain biopsies, otherwise an increase in mortality may result if empirical treatment and biopsy are both delayed. A recent study found that the median delay in beginning pharmacotherapy in cases of ring-enhancing brain lesions is 2 days ${ }^{[5]}$, which may still be too long in severe cases.

\section{REFERENCES}

1. Mahato PS, Dabhi AS, Thorat PB. Clinical and investigative profile of ring-enhancing lesions on neuroimaging. Indian Journal of Clinical Practice 2012;22:512-518.

2. Garg R, Sinha M. Multiple ring-enhancing lesions of the brain. J Postgrad Med 2010;56:307.

3. Chen S, Pu J-L, Yu J, Zhang J-M. Multiple Aspergillus cerebellar abscesses in a middle-aged female: case report and literature review. Int J Med Sci 2011;8:635-639.

4. Sessa A, Meroni M, Battini G, et al. Nosocomial outbreak of Aspergillus fumigatus infection among patients in a renal unit? Nephrol Dial Transpl 1996;11:1322-1324.

5. Gutiérrez-Cuadra M, Ballesteros-Sanz MA, Vallejo A, Miñambres E, Fariñas Alvarez C, García-Palomo JD, García-Palomo JD, et al. Brain abscess in a tertiary medical center: epidemiology and prognostic factors related to mortality. Rev Española Quimioter 2009;22:201-206. 